\title{
Worst-Case Routing Performance Evaluation of Sensor Networks
}

\author{
Edwin Soedarmadji \\ California Institute of Technology \\ Pasadena, CA 91125, USA \\ edwin@systems.caltech.edu
}

\begin{abstract}
Successful sensor network applications depends heavily on the ability of these networks to reliably and reasonably perform under the worst-case scenarios, extreme and unusual events for which many such networks are designed to detect. One of the key performance measures is the network's ability to route measurement data from the sensor nodes to the destination node(s). This paper introduces a general framework with which worst-case routing performance of different sensor networks can be evaluated and compared. Our method can either be used as a design optimization tool, or a decision making tool to select and price contending sensor network designs and applications.
\end{abstract}

\section{Introduction}

Sensor networks have steadily become parts of everyday life: traffic sensors, fire alarms, and motion detectors are now connected to form large networks in many buildings, factories, and facilities to maintain security, environmental control, and track valuable assets.

Sensor networks are poised for integration into many current and future mission critical systems. Examples of such mission critical systems already exist today: the Advanced National Seismic System (ANSS) [1] and the Pacific Tsunami Warning System operated by the National Weather Service [2] are two large networks of sensors designed to reliably detect, measure, report, and track large scale, catastrophic geological events moving at the speed of several kilometers per second. Similarly, sensor networks can be used to detect the presence of dangerous levels of biohazards, radiation, lethal mineshaft gases, etc.

In these applications, the network has to be designed such that the probability of each sensor successfully transmitting information to the base node is maximized since losing even one symbol, packet, or file can have very disastrous consequences. Retransmission might be impossible, as the originating sensors themselves could have been destroyed.
Fault tolerance is therefore a highly desired property of any sensor network design. Among the routing algorithms reviewed in $[3,4]$, many are designed to be fault-tolerant [5-10]. However, obviously their worst-case fault tolerance ultimately depends on the network design used. Given two or more competing sensor network designs, which one would best meet the worst-case routing performance requirements demanded by mission critical applications?

This problem motivates us to introduce a worst-case routing performance metric to measure and compare network path "lengths" that depend on the paths' worst-case probability of failure. Under ideal conditions, the path that minimizes this length is selected for data transmission between a source-destination pair. The minimum (or best) path length over all source-destination pairs can then be used to evaluate the network's worst case routing performance. We call this metric the Worst-Case Error (WCE) metric, where an "Error" can represent any type of failure.

Just as in any path metric, a WCE path's length depends on its edges' lengths. However, in particular, the WCE length depends on how the failure events on each network link is probabilistically modeled and parametrized. This paper provides a general condition for such models to be compatible for WCE analysis, and uses as specific examples three network models where each link is modeled as a $q$-ary Symmetric Channel (q-SC), a $q$-ary Erasure Channel, and a non-negative mean AWGN channel. These channels are parametrized by their symbol error ratio, symbol erasure ratio, and the mean-variance pair. For convenience, we consider these parameters as edge metrics referred to as the "Bit Error Ratio" (BER) — which is quite a misnomer because our model is not restricted to binary communication channels, and the parameter is not always a ratio.

The method presented in this paper is applicable to many other worst-case optimization problems. Although here we use the WCE metric for evaluating sensor networks, it can be used in other network problems that attempt to minimize the worst-case occurence probability of non-typical, but highly catastrophic data transmission failures. 


\section{Formulation and Notation}

The network is modeled with a digraph $G=(V, E)$, where $V, E$, and $\Pi$ are the node, edge and path sets of $G$. Two nodes $s$ and $d \in V$ are the source and destination nodes, and $\Pi \subset \Pi$ denotes the set of all paths from $s$ to $d$.

A path $\pi \in \Pi$ whose nodes $V_{\pi} \subset V$ are connected by $E_{\pi} \subset E$ is denoted by either $\left\langle v_{0}, \ldots, v_{J}\right\rangle,\left\langle e_{1}, \ldots, e_{J}\right\rangle$, or $\left\langle v_{0}, e_{1}, \ldots, e_{J}, v_{J}\right\rangle$. The symbol $\left\langle v_{i}, v_{i+1}\right\rangle$ denotes the edge (path) connecting the two (non-) adjacent nodes $v_{i}$ and $v_{i+1}$. A partial path $\pi_{j}$ of $\pi$ denotes $\left\langle v_{0}, \ldots, v_{j}\right\rangle$, with $0<$ $j \leq J$, and a truncated path $\bar{\pi}_{j}$ is $\left\langle v_{0}, e_{1}, \ldots, v_{j-1}, e_{j}\right\rangle$.

Denote the message by $B \in \mathcal{B}$, where $\mathcal{B}$ is the space of all allowable messages in the network. Let $B_{i}$ denote the value of $B$ as it departs from $v_{i}$; and let $\bar{B}_{i}$ denote the value of $B$ as it leaves $e_{i}$. Both $v_{i}$ and $e_{i}$ are parts of $\left\langle v_{0}, e_{1}, \ldots, e_{J}, v_{J}\right\rangle$, along which $B$ evolves as follows:

$$
B_{0} \stackrel{e_{1}}{\longrightarrow} \bar{B}_{1} \stackrel{v_{1}}{\longrightarrow} B_{1} \stackrel{e_{2}}{\longrightarrow} \bar{B}_{2} \stackrel{v_{2}}{\longrightarrow} \cdots \stackrel{e_{J}}{\longrightarrow} \bar{B}_{J} \stackrel{v_{J}}{\longrightarrow} B_{J}
$$

where $v_{i}$ and $e_{i}$ correspond to the operators $\boldsymbol{v}_{i}, \boldsymbol{e}_{i} \in \mathcal{E}$ : $\mathcal{B} \rightarrow \mathcal{B}$ given by $B_{i}=\boldsymbol{v}_{i}\left(\bar{B}_{i}\right)$ and $\bar{B}_{i+1}=e_{i}\left(B_{i}\right)$. The operator for $\pi$ is $\boldsymbol{\pi}=\boldsymbol{v}_{J} \circ \boldsymbol{e}_{J} \circ \cdots \circ \boldsymbol{e}_{1} \circ \boldsymbol{v}_{0}$. For $\pi_{j}$, it is $\boldsymbol{\pi}_{j}=\boldsymbol{v}_{j} \circ \boldsymbol{e}_{j} \circ \cdots \circ \boldsymbol{e}_{1} \circ \boldsymbol{v}_{0}$, and for $\bar{\pi}_{j}$, it is $\overline{\boldsymbol{\pi}}_{j}=\boldsymbol{e}_{j} \circ$ $\boldsymbol{v}_{j-1} \cdots \circ \boldsymbol{e}_{1} \circ \boldsymbol{v}_{0}$. Thus, $\boldsymbol{\pi}_{j}\left(B_{0}\right)=B_{j}$ and $\overline{\boldsymbol{\pi}}_{j}\left(B_{0}\right)=\bar{B}_{j}$.

Define the function $X: \mathcal{B} \times \mathcal{B} \rightarrow \mathcal{M}$ that measures the distance $x \in \mathcal{M}$ between a message $B_{0}$ at $v_{0}$ which evolves into $B_{J}$ at $v_{J}$, where $v_{0}$ and $v_{J}$ are connected by $\left\langle v_{0}, v_{J}\right\rangle$, and $\mathcal{M}$ is the metric space. The distance $x$ from $B_{i}$ to $B_{i^{\prime}}$ is denoted by $x=X_{i, i^{\prime}}=X\left(B_{i}, B_{i^{\prime}}\right)$, and $X_{i, \bar{i}^{\prime}}$ is a shorthand for $X\left(B_{i}, \bar{B}_{i^{\prime}}\right)$. If $e=\left\langle v_{i}, v_{i^{\prime}}\right\rangle$ then we define the shorthand $X(\epsilon)=X\left(B_{i}, \bar{B}_{i^{\prime}}\right)$.

Most often, $X_{0, i}$ are random variables. Consider the random variable $x=X_{i, i^{\prime}}$ that measures the distance between the message at $v_{i}$ and its image at $v_{i^{\prime}}$. Define $P(X=x, \boldsymbol{\lambda})$ as the probability density parametrized by a vector $\lambda \in \Lambda$. The value $\boldsymbol{\lambda}=\infty$ denotes the absence of connection between two nodes. Each edge $e_{i} \in E$ has its own $\boldsymbol{\lambda}_{i}$

Finally, we define the worst case function $\bar{x}\left(\boldsymbol{\lambda}_{i}, \epsilon\right)$ that computes, given a density $P(x, \boldsymbol{\lambda})=P\left(X=x, \boldsymbol{\lambda}_{i}\right)$, the worst case value of $x$ defined as follows:

$$
\bar{x}\left(\boldsymbol{\lambda}_{i}, \epsilon\right)=\max _{x}\left\{x \mid P\left(X>x, \boldsymbol{\lambda}_{i}\right) \geq \epsilon, x \in \mathcal{M}\right\}
$$

Consider a path $\pi=\left\langle e_{1}, \ldots, e_{J}\right\rangle \in \Pi$ and its partial path $\pi_{j}=\left\langle e_{1}, \ldots, e_{j}\right\rangle$ with $1 \leq j \leq J$. For convenience, we also define the function $\beta: \Pi \rightarrow \Lambda$ that maps a path $\pi$ (or an edge $e_{i}$ ) into a density parameter $\boldsymbol{\lambda}_{\pi}$ (or $\boldsymbol{\lambda}_{i}$ ) and the function $\omega: \Pi \rightarrow \mathcal{M}$ that maps a path or an edge (given $\epsilon$ ) into its worst case value $x \in \mathcal{M}$. For $e_{i}$, the $\beta$ and $\omega$ are related to $\bar{x}\left(\boldsymbol{\lambda}_{i}, \epsilon\right)$ through: $\omega\left(e_{i}\right)=\bar{x}\left(\beta\left(e_{i}\right), \epsilon\right)$. For $\pi$, assuming $\lambda_{\pi}$ is defined, similarly we have $\omega(\pi)=\bar{x}(\beta(\pi), \epsilon)$. The next question is, how does $\boldsymbol{\lambda}_{\pi}$ depend on $\boldsymbol{\lambda}_{i}$ 's, and how does $\bar{x}\left(\boldsymbol{\lambda}_{\pi}, \epsilon\right)$ depend on $\bar{x}_{i}=\bar{x}\left(\boldsymbol{\lambda}_{i}, \epsilon\right)$ ?
Let us assume that the addition operation is defined in $\Lambda$ and $\mathcal{M}$ and is denoted by $\oplus$. If $x_{1}=X\left(e_{1}\right), x_{2}=X\left(e_{2}\right)$, $\lambda_{1}=\beta\left(e_{1}\right), \lambda_{2}=\beta\left(e_{2}\right), \bar{x}_{1}=\omega\left(e_{1}\right), \bar{x}_{2}=\omega\left(e_{2}\right)$, and $\pi=\left\langle e_{1}, e_{2}\right\rangle$, then we say $x_{\pi}=x_{1} \oplus x_{2}, \boldsymbol{\lambda}_{\pi}=\boldsymbol{\lambda}_{1} \oplus \boldsymbol{\lambda}_{2}$, or $\bar{x}_{\pi}=\bar{x}_{1} \oplus \bar{x}_{2}$. For these expressions to make sense, the $\oplus$, $\Lambda$ and $\mathcal{M}$ have to obey the properties in the next section.

With $\oplus$, we can now define $x_{\pi}, \boldsymbol{\lambda}_{\pi}$ and $\bar{x}_{\pi}$ in terms of $x_{i}, \boldsymbol{\lambda}_{i}$ and $\bar{x}_{i}$ using a generalized summation: $x_{\pi}=\bigoplus x_{i}$, $\boldsymbol{\lambda}_{\pi}=\bigoplus \boldsymbol{\lambda}_{i}$ and $\bar{x}_{\pi}=\bigoplus \bar{x}_{i}$. The pairings of $\Lambda$ and $\mathcal{M}$ with $\oplus$ form what we call the $\mathbf{X}, \mathbf{B}$, and $\mathbf{W}$ algebras, from the $X, \beta$, and $\omega$ functions, respectively.

Between two nodes, the optimal path $\pi^{*}$ is the path with the "shortest" path length from $s$ to $d$ when measured in the $\mathbf{X}, \mathbf{B}$ or $\mathbf{W}$ algebra (or metric). However, having $\oplus, X, \beta$, and $\omega$ is not enough to calculate $\pi^{*}$. We need to compare path lengths. Therefore we need a total order $\preceq$ on $\Lambda$ and $\mathcal{M}$ to evaluate expressions like $x_{\pi} \preceq x_{\pi^{\prime}}, \boldsymbol{\lambda}_{\pi} \preceq \boldsymbol{\lambda}_{\pi^{\prime}}$, or $\bar{x}_{\pi} \preceq \bar{x}_{\pi^{\prime}}$. Once $\preceq$ is defined, then we have:

$$
\begin{aligned}
& x^{*}=\min _{\pi}\left\{x_{\pi} \mid \pi \in \Pi\right\} \\
& \lambda^{*}=\min _{\pi}\left\{\boldsymbol{\lambda}_{\pi} \mid \pi \in \Pi\right\} \\
& \bar{x}^{*}=\min _{\pi}\left\{\bar{x}_{\pi}=\bar{x}\left(\boldsymbol{\lambda}_{\pi}, \epsilon\right) \mid \pi \in \Pi\right\}
\end{aligned}
$$

Example 1 ( $q$-ary Symmetric Channels) Let $Q$ be the $q$ ary alphabet $\{0, \ldots, q-1\}$. Suppose that the source $s$ produces $n$-symbol messages $B \in Q^{n}$, with $B_{l} \in Q$, and $l=1 \ldots n$. Each network link is modeled as a $q$-ary Symmetric Channel (q-SC) with symbol error rate $p$.

Let $B_{i}$ (and $b_{i}=B_{l i}$ ) denote $B$ (and $B_{l}$ ) as it departs from $v_{i}$; and let $\bar{B}_{i}$ (and $\bar{b}_{i}=\bar{B}_{l i}$ ) denote $B$ (and $B_{l}$ ) as it leaves $e_{i}$. The transition probability defining a q-SC is:

$$
P\left(b_{i+1} \mid b_{i}\right)= \begin{cases}1-p & , b_{i}=b_{i+1} \\ p /(q-1) & , b_{i} \neq b_{i+1} .\end{cases}
$$

The operators $\boldsymbol{v}_{i}, \boldsymbol{e}_{i} \in \mathcal{E}: Q^{n} \rightarrow Q^{n}$ given by $B_{i}=$ $\boldsymbol{v}_{i}\left(\bar{B}_{i}\right)$ and $\bar{B}_{i+1}=\boldsymbol{e}_{i}\left(B_{i}\right)$. The function $X$ measures the number of errors (Hamming distance) in $B_{i}$ compared to $B_{i^{\prime}}$, denoted by $x=X_{i, i^{\prime}}=X\left(B_{i}, B_{i^{\prime}}\right)=\mid\left\{l \mid b_{i} \neq\right.$ $\left.b_{i^{\prime}}\right\} \mid$. In a q-SC, the scalar parameter that plays the role of $\lambda$ is $p$. The density $P(x, \boldsymbol{\lambda})=P(x, p)$ is:

$$
P(x, p)= \begin{cases}\left(\begin{array}{l}
n \\
x
\end{array}\right) p^{x}(1-p)^{n-x} & , p \in(0,1) \\
\delta(x) & , p=0 \\
\delta(x-n) & , p=1, \infty\end{cases}
$$

The $\mathbf{B}$ and $\mathbf{W}$ algebras are such that two adjacent edges $e_{1}$ and $e_{2}$ with parameters $\boldsymbol{\lambda}_{1}$ and $\boldsymbol{\lambda}_{2}$ can be viewed as a single edge with parameter $\boldsymbol{\lambda}=\boldsymbol{\lambda}_{1} \oplus \boldsymbol{\lambda}_{2}=p_{1} \oplus p_{2}$ defined by:

$$
\begin{aligned}
& p_{1} \oplus p_{2}=1-\left(1-p_{1}\right)\left(1-p_{2}\right)-\left(p_{1} p_{2}\right) /(q-1) \\
& \bar{x}_{1} \oplus \bar{x}_{2}=\max \left\{x \mid P\left(x, p_{1} \oplus p_{2}\right) \geq \epsilon\right\}
\end{aligned}
$$

In event- and packet-based scenarios, symbol alphabets (and the probabilities) correspond to event or packet states. 
Example 2 ( $q$-ary Erasure Channels) The symbol $q-1$ in $Q$ is designated as a special erasure symbol. Each network link is modeled as a $q$-ary Erasure Channel (q-EC) with symbol erasure rate $p$. The transition probability is:

$$
P\left(b_{i+1} \mid b_{i}\right)= \begin{cases}1-p & , b_{i}=b_{i+1} \\ p & , b_{i} \neq b_{i+1}=q-1 .\end{cases}
$$

The function $X$ measures the number of erasures in $B_{i}$ relative to $B_{i^{\prime}}$, denoted by $x=X_{i, i^{\prime}}=X\left(B_{i}, B_{i^{\prime}}\right)=$ $\left|\left\{l \mid b_{i} \neq b_{i^{\prime}}=q-1\right\}\right|$. The probability density function $P(x, \boldsymbol{\lambda})$ is the same as in Example 1, except $p$ is the link symbol erasure probability and the $\mathbf{B}$ algebra is $\boldsymbol{\lambda}=\boldsymbol{\lambda}_{1} \oplus \boldsymbol{\lambda}_{2}=p_{1} \oplus p_{2}$ defined by:

$$
p_{1} \oplus p_{2}=1-\left(1-p_{1}\right)\left(1-p_{2}\right)
$$

$B$ can be defined as a block with $n$ packets of $m$ symbols each, and erasures represent lost packets.

Example 3 (Non-negative-mean AWGN) In this example, the message is a scalar $B \in \mathbb{R}^{+}$with non-negativemean AWGN on each link. This message represents the amount of degradation a measurement value has experienced. Therefore, the source always transmits $B_{0}=0$.

The AWGN is characterized by $\boldsymbol{\lambda}=\left(\mu \geq 0, \sigma^{2}\right)$, where $\mu$ is the mean, and $\sigma^{2}$ the variance of the Gaussian density and the transition probability density $P\left(B_{i+1} \mid B_{i}\right)$ is:

$$
P\left(x ; \mu, \sigma^{2}\right)=\frac{1}{\sqrt{2 \pi \sigma^{2}}} \exp \left(\frac{-(x-\mu)^{2}}{2 \sigma^{2}}\right)
$$

where $x=B_{i+1}-B_{i}$. The definition for the $\mathbf{B}$ algebra is derived from the fact that any two independent, adjacent edges $e_{1}$ and $e_{2}$ with parameters $\boldsymbol{\lambda}_{1}$ and $\boldsymbol{\lambda}_{2}$ can be treated as a single edge with parameter $\boldsymbol{\lambda}=\boldsymbol{\lambda}_{1} \oplus \boldsymbol{\lambda}_{2}=\boldsymbol{\lambda}_{1}+\boldsymbol{\lambda}_{2}$, where the + sign is the standard vector summation operator. Since $x$ is continuous, $\bar{x}=F^{-1}\left(\epsilon ; \mu, \sigma^{2}>0\right)$ can be obtained analytically by solving the following equation:

$$
\begin{aligned}
F\left(\bar{x} ; \mu, \sigma^{2}\right) & =P\left(X>x ; \mu, \sigma^{2}\right) \\
& =\frac{1}{2} \operatorname{erfc}\left(\frac{\bar{x}-\mu}{\sqrt{2 \sigma^{2}}}\right)=\epsilon
\end{aligned}
$$

From this result, we can also define the $\mathbf{W}$ algebra as follow:

$$
\bar{x}_{1} \oplus \bar{x}_{2}=F^{-1}\left(\epsilon ; \mu_{1}+\mu_{2}, \sigma_{1}^{2}+\sigma_{2}^{2}\right) .
$$

It is useful to think of $\lambda$ 's as $2 \mathrm{D}$ vectors in the positive octant $\Lambda=\mathbb{R}^{+2}$, and the function $\bar{x}$ as an element in $\overline{\mathcal{X}}_{\epsilon}: \Lambda \rightarrow \mathbb{R}$, defining isocontours for each value of $\bar{x}$.

It is also interesting to note that at $\sigma^{2}=0$, the value $\bar{x}$ reaches its limit of $\mu$. In addition, we can also compute $G$ 's maximum path variance $\sigma_{\text {max }}^{2}$ by using the longest path algorithm on $G$ with edge metrics $\sigma_{i}^{2}$ and usual addition operator [11]. Next, we show how the density parameters from the above examples can be used as routing metrics.

\section{Generalized Dijkstra's Algorithm}

The problem of finding the optimal path that minimizes the metric of choice ( $x, \lambda, \bar{x}$, or others) in a DTN that is represented by a graph $G$ can be solved using the Generalized Dijkstra's Algorithm (GDA) below [12]:

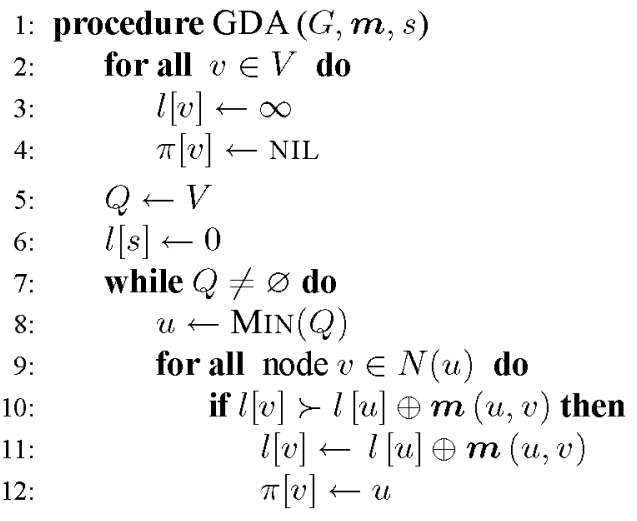

The GDA is practically identical to the Dijkstra's Algorithm (DA) except for the relaxation step, where $\oplus$ and $\preceq$ operators act on a general metric space $\mathcal{M}$ (instead of the equivalent step in DA, where + and $\leq$ operators act on $\mathbb{R}$ ).

On line $9, N(u)$ denotes the set of all nodes adjacent to $u$. The argument $m$ is the BER lengths of the edges in $G$ each of which is an element in $\mathcal{M}$, and $\boldsymbol{m}(u, v)$ is the BER length of $\langle u, v\rangle$. Lines 10-12 perform the relaxation step of the GDA. This step depends on the definitions of $\mathcal{M}, \oplus$, and $\preceq$. If the GDA (in)correctly returns the path in $G$ with minimum length measured in $\mathcal{M}$, then $(\mathcal{M}, \oplus)$ and $\preceq$ are said to be (in) compatible with the GDA. The following is the required properties for compatibility:

Proposition 1 An algebra $\mathbf{A}=(\mathcal{M}, \oplus)$ and a total order $\preceq$ is compatible with the GDA if and only if it satisfies all the properties in the set denoted by $\mathbf{P}$ below:

P1 is a commutative monoid, that is, for $a, b, c \in \mathcal{M}$ :

- $\mathcal{M}$ is closed under $\oplus: a \oplus b \in \mathcal{M}$;

- $\oplus$ is associative : $a \oplus(b \oplus c)=(a \oplus b) \oplus c$;

- 0 is the identity : $a \oplus 0=0 \oplus a=a$;

- $\oplus$ is commutative : $a \oplus b=b \oplus a$.

P2 There exists $\infty \in \mathcal{M} \mid a \oplus \infty=\infty \oplus a=\infty$.

$\mathbf{P 3} \preceq$ is a total order on $\mathcal{M}$, i.e., $\preceq$ is :

- reflexive: $a \preceq a$;

- anti-symmetric: if $a \preceq b$ and $b \preceq$ a then $a=b$;

- transitive: if $a \preceq b$ and $b \preceq c$ then $a \preceq c$;

- total: for every $a, b \in \mathcal{M}$ either $a \preceq b$ or $b \preceq a$.

P4 There exists the least element 0 that satisfies $0 \preceq a$.

P5 $a \oplus c \prec b \oplus c$ if $a \prec b$ and $c \in \mathcal{M}-\{\infty\}$.

PROOF: Refer to [12] for a complete proof.

Next, we prove that the $\mathbf{B}$ algebra from examples 1-3 are compatible with the GDA. 
Theorem 2 The algebras $\mathbf{B}=(\Lambda, \oplus)$ defined in examples $1-3$ and their respective total orders $\preceq$ satisfy all the properties in $\mathbf{P}$, and thus compatible with the GDA.

Proof: The proofs for q-SC and q-EC are omitted because they are mostly algebraic. For AWGN, since $\Lambda=$ $\mathbb{R}^{2+}$ and $\mathbb{R}^{2+}$ is not a totally ordered set, we must define $\preceq$ in terms of $\bar{x}(\boldsymbol{\lambda}, \epsilon)$ as follows:

$$
\begin{aligned}
\boldsymbol{\lambda} \preceq \boldsymbol{\lambda}^{\prime} \Leftrightarrow \bar{x}(\boldsymbol{\lambda}, \epsilon) & <\bar{x}\left(\boldsymbol{\lambda}^{\prime}, \epsilon\right) \text { or } \\
\bar{x}(\boldsymbol{\lambda}, \epsilon) & =\bar{x}\left(\boldsymbol{\lambda}^{\prime}, \epsilon\right) \text { and } \sigma^{2} \leq \sigma^{2}
\end{aligned}
$$

The space $\Lambda$ is depicted in figure 1. First, the values $\lambda$ 's are sorted by their $\bar{x}$ contour. Along each $\bar{x}$ contour, the values $\lambda$ are ordered from lower values of $\sigma^{2}$ to the higher ones.

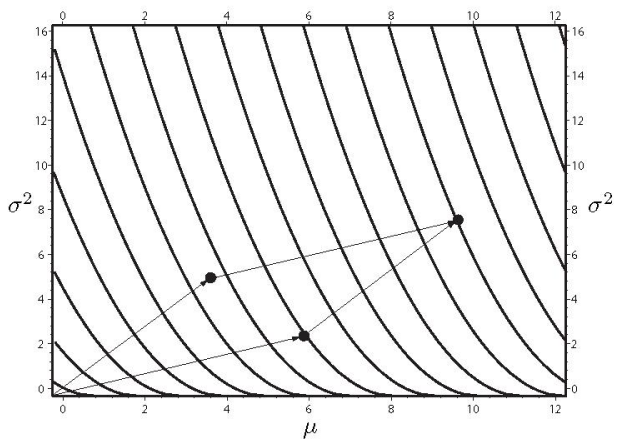

Figure 1: The metric space $\Lambda$.

P1 Closure obviously follows from the vector addition properties of $\lambda$. In case one (or both) of the operands is $\infty$, then by definition, the $\oplus$ sum is also $\infty \in \Lambda$.

P2 The proof is derived from closure on $\infty$.

P3 The proof follows from the definition of $\Lambda$ and $\preceq$.

P4 The function $F$ in equation (9) is minimized when $\boldsymbol{\lambda}=(0,0) \in \Lambda$, and is thus the 0 element.

P5 The proof is obvious from figure 1, the definition of $\Lambda$, and concavity of the function $\mu\left(\sigma^{2}\right)$.

Theorem 3 The $\bar{x}$ values in examples $1-3$ are nondecreasing functions of their respective $\lambda$ 's, which means that the path with minimum $\lambda$ obtained from the GDA is also the path with minimum $\bar{x}$.

PROOF: The proof for AWGN automatically follows from the definition of $\boldsymbol{\lambda} \preceq \lambda^{\prime}$ and is omitted. For q-SC (and q-EC, which is identical) we prove the theorem for a binomial distribution with $\boldsymbol{\lambda}=(n, p)$. From [13] we have:

$$
\begin{aligned}
P(X>x, p) & =\sum_{X=x+1}^{n}\left(\begin{array}{l}
n \\
X
\end{array}\right) p^{X}(1-p)^{n-X} \\
& =I_{p}(x+1, n-x)
\end{aligned}
$$

Where $I_{p}(x+1, n-x)$ is the regularized incomplete Beta function, taking two non-negative integer arguments.
This curious function in turn is defined as the ratio of an incomplete Beta function $B_{p}(x+1, n-x)$ and a regular Beta function $B(x+1, n-x)$, from which equation the term "regularized" sounds quite similar to "normalized":

$$
\begin{aligned}
& I_{p}(x+1, n-x)=\frac{B_{p}(x+1, n-x)}{B(x+1, n-x)} \\
& =B_{p}(x+1, n-x) \times \frac{n !}{x !(n-x-1) !} \\
& =(n-x)\left(\begin{array}{l}
n \\
x
\end{array}\right) \int_{0}^{p} t^{x}(1-t)^{n-x-1} d t .
\end{aligned}
$$

Since $n$ is constant over the network, for each $0 \leq x \leq n$, the function $I_{p}(x+1, n-x)$ is really just a function of $p$. Intuitively, it is obvious that $I_{p}$ is a non-decreasing function of $p$. We can prove this by taking the first derivative with respect to $p$ for fixed $n$ and $x$ :

$$
\begin{aligned}
\frac{\partial I_{p}}{\partial p} & =(n-x)\left(\begin{array}{l}
n \\
x
\end{array}\right) \frac{\partial}{\partial p} \int_{0}^{p} t^{x}(1-t)^{n-x-1} d t \\
& =(n-x)\left(\begin{array}{l}
n \\
x
\end{array}\right) p^{x}(1-p)^{n-x-1} \geq 0
\end{aligned}
$$

because $x \leq n$ and $0 \leq p \leq 1$. This means for a given $n$ and $x$, as we increase $p$, the value of $P(X>x, p)=I_{p}(x+$ $1, n-x)$ is non-decreasing. It is important to note that for $0 \leq x<n$, if $p=0$, then the function $P(X>x, p)=0$, while if $p=1$, then $P(X>x, p)=1$.

For intermediate values of $0<p<1$, the set of values $\boldsymbol{P}=\left\{P_{0}, P_{1}, \ldots, P_{n+1}\right\}$ of $P(X>x, p)$ at $x=$ $0,1, \ldots, n-1$ are non-increasing as $x$ increases.

Recall the equation defining $\bar{x}$ as a function of $\epsilon$ and , which is adapted for $\boldsymbol{\lambda}=p$ and $\mathcal{M}=[0, n-1] \subset \mathbb{N}$.

$$
\bar{x}(p, \epsilon)=\max _{x}\{x \mid P(X>x, p) \geq \epsilon, 0 \leq x<n\}
$$

This equation partitions the set $\boldsymbol{P}$ into $\boldsymbol{P}^{+}=\left\{P_{i} \mid P_{i} \geq\right.$ $\left.\epsilon, P_{i} \in \boldsymbol{P}\right\}$, i.e., the values $P_{i}$ greater than $\epsilon$, and its complement $\boldsymbol{P}^{-}=\left\{P_{i} \mid P_{i}<\epsilon, P_{i} \in \boldsymbol{P}\right\}$. We can define the corresponding index sets $\mathcal{M}^{+}=\left\{i \mid P_{i} \in \boldsymbol{P}^{+}, i \in \mathcal{M}\right\}$ and $\mathcal{M}^{-}=\left\{i \mid P_{i} \in \boldsymbol{P}^{-}, i \in \mathcal{M}\right\}$. Thus, we can view $\bar{x}(p, \epsilon)$ as the maximum value $i \in \mathcal{M}^{+}$bordering $\mathcal{M}^{-}$.

$$
\bar{x}(p, \epsilon)=\max \left\{i \mid i \in \mathcal{M}^{+}\right\}
$$

However, from equation (14), the values $P_{i}$ are nondecreasing functions of $p$ (while simultaneously nonincreasing with respect to $i$ for each value of $p$ ).

Consequently, as $p$ increases, the cardinality $\left|\boldsymbol{P}^{+}\right|$of $\boldsymbol{P}$ increases. Since $\left|\mathcal{M}^{+}\right|=\left|\boldsymbol{P}^{+}\right|$, so does the cardinality of $\mathcal{M}^{+}$. However, since both $\mathcal{M}^{+}$and $\mathcal{M}^{-}$are two contiguous partitions of $\mathcal{M}$, this implies $\bar{x}$ (the maximum element of $\mathcal{M}^{+}$) must increase as a function of $p$. 


\section{Conclusion and Discussion}

The preceding results provide us with a practical method to measure the worst-case routing performance of a sensor network. The method is based on the Generalized Dijkstra's Algorithm (GDA), which requires the edge metric to obey a certain set of necessary and sufficient conditions. Along each path, the maximum worst-case failure probability is computed. GDA then minimizes the maximum probability over all network path.

We provide three examples where the links are modeled as $q$-ary symmetric channels, $q$-ary erasure channels, and non-negative mean AWGN channels, each with its own edge metric that is nothing more than the parameters of its transmission failure probability density.

By letting the metric to be density parameters, we compute any path length by an appropriate combination of its edge lengths according to the laws of probability, thus fully preserving the stochastic nature of the routing problem (instead of simply reducing the stochastic edge weights to proxy deterministic values). For a given level of "possibility threshold" $\epsilon$, each edge density corresponds to a unique worst case value $\bar{x}$. We showed that edge worst case value can be combined into path worst case values, and that the minimum $\bar{x}^{*}$ of such values over a network can be computed using the GDA.

The pair $\bar{x}^{*}$ and $\epsilon$ allow us to compare the worst-case network routing performance. Given a constant benchmark worst-case value $\bar{x}$ for all the networks $\left\{G_{i}\right\}$ under evaluation, we can solve for the $\epsilon_{i}$ value for each network $G_{i}$. The network $G^{*}$ with the lowest value of $\epsilon^{*}$ is the network with the best worst-case routing performance. Alternatively, the $\epsilon$ value can be fixed for all the networks under evaluation. The worst-case values $\bar{x}_{i}$ can then be compared to select the network $G^{*}$ with the best worst-case performance, i.e., the smallest failure rate $\bar{x}_{i}$ among all the networks $G_{i}$.

Future work includes simulating or experimentally verifying the theoretical results presented herein, and generalizing the results further to other types of edge weight densities. Our preliminary work indicated that these extensions are highly feasible. Another interesting application would be to use the worst-case performance evaluation method on actual or planned mission critical sensor network projects.

\section{Acknowledgement}

The author would like to convey his appreciation to Prof. Robert J. McEliece for valuable discussions and guidance, as well as useful suggestions that have improved this manuscript. Financial support from the Caltech Lee Center for Advanced Networking and NSF Grant No. CCF0514881 are gratefully acknowledged.

\section{References}

[1] "ANSS - Advanced National Seismic System," web site address at http://earthquake.usgs.gov/anss/.

[2] "PTWC - Pacific Tsunami Warning Center," web site address at http://www.prh.noaa.gov/pr/ptwc/.

[3] E. Royer and C. Toh, "A review of current routing protocols for ad-hoc mobile wireless networks," IEEE Personal Communications Magazine, vol. 6, no. 2, pp. 46-55, April 1999.

[4] A. Ahmed, H. Shi, and Y. Shang, "A survey on network protocols for wireless sensor networks," Proceeding of the International Conference on Information Technology, pp. 301-305, August 2003.

[5] C. Intanagonwiwat, R. Govindan, and D. Estrin, "Direct diffusion: a scalable and robust communication paradigm for sensor networks," Proceedings of MobiCom, August, 2000.

[6] W. Heinzelman, A. Chandrakasan, and H. Balakrishnan, "An application-specific protocol architecture for wireless microsensor networks," IEEE Transactions on Wireless Communications, vol. 1, no. 4, pp. 660670, October 2002.

[7] B. Karp and H. T. Kung, "GPSR: greedy perimeter stateless routing for wireless networks," Proceedings of the MobiCom, August, 2000.

[8] Y. Xue and K. Nahrstedt, "Fault Tolerant Routing in Mobile Ad Hoc Networks," Proceedings of IEEE Wireless Communication and Networking Conference, pp. 1174-1179, March, 2003.

[9] V. Park and M. Corson, "A highly adaptive distributed routing algorithm for mobile wireless networks," Proceedings of INFOCOM'97, pp. 1405-1413, April 1997.

[10] Huang X., Deng J., Ma J., and Wu Z., "Fault Tolerant Routing For Wireless Sensor Grid Networks", Proceedings of IEEE Sensors Applications Symposium 2006, February 2006.

[11] Cormen, T.H., et al., Introduction to Algorithms, MIT Press, MA and McGraw-Hill, NY, 1990.

[12] Sobrinho, J.L., "Algebra and algorithms for QoS path computation and hop-by-hop routing in the Internet," IEEE/ACM ToN., vol. 10, pp. 541-550, August 2002.

[13] Abramowitz M., Stegun I., Handbook of mathematical functions, National Bureau of Standards, Applied Mathematics Series - 55, December 1972. 